\section{Interview mit dem Kongresspräsidenten Herrn Prof. Hermann Helmberger}

\author{
In Hamburg findet dieses Jahr der 93. Röntgenkongress unter \\ dem Motto „Gegensätze verbinden“ statt. Der Kongress- \\ präsident Prof. Hermann Helmberger gibt im Interview Einbli- \\ cke, was die Besucher erwartet und was auf keinen Fall ver- \\ säumt werden darf.
}

? Herr Professor Helmberger, verbinden Gegensätze tatsächlich?

Helmberger: Für diese 1. Frage bin ich Ihnen wirklich sehr dankbar, denn sie zeigt mir doch, dass das Kongressmotto richtig gewählt war. Wir hatten ganz bewusst auf jegliche Interpunktion verzichtet, um so noch Platz für die eigene Interpretation des Lesenden oder Hörenden und die Diskussion dazu zu lassen. Die Radiologie ist ein Querschnittsfach und hat eine Breite der Themen erreicht, die sich in manchen Bereichen scheinbar zu widersprechen scheinen, d.h. thematisch oder aufgrund der versorgten Patientengruppen oder auch bezüglich der Herangehensweise. Mir war es sehr wichtig in der Vorbereitung des Kongresses, hier Gemeinsamkeiten wieder mehr in den Vordergrund $z u$ rücken und zu betonen.

Deshalb wird es auch gemeinsame Veranstaltungen z.B. der AG-Herz-Diagnostik und der DEGIR oder der AGs Uro-Radiologie und Kinderradiologie geben. Neben diesen Gemeinsamkeiten - innerhalb der Radiologie, die es zu betonen gilt - trifft dies aber auch auf benachbarte Fächer der Bildgebung, z.B. die Nuklearmedizin und die Kinderradiologie, zu. Auch hier werden wir uns beim Kongress den Fachthemen gemeinsam nähern.

Und der 3. Schritt geht über das Gebiet der Bildgebung hinaus: die interdisziplinäre Diskussion mit den klinischen Fachkollegen, an deren Patientenversorgung wir beteiligt sind. Und so freue ich mich besonders, dass wir insgesamt 8 klinische Fachgesellschaften mit ihren Vertreten, in unser Programm einbinden konnten und gemeinsam aktuelle Fragen erörtern werden.

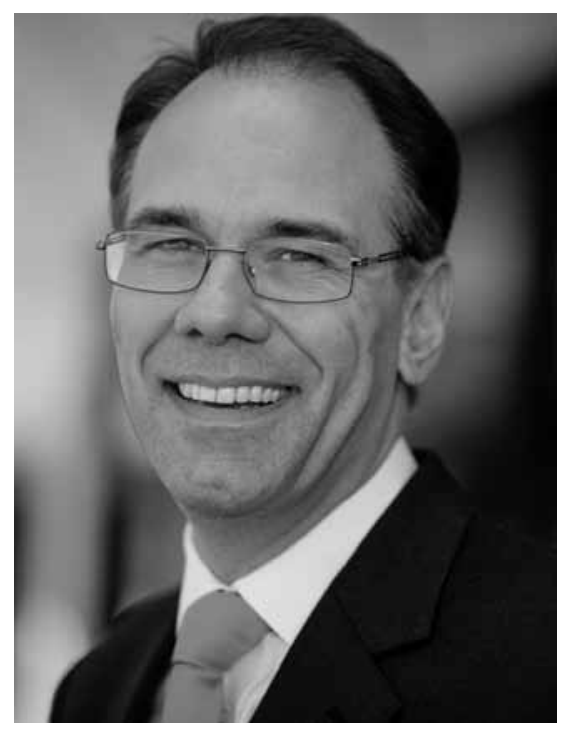

? Welche Themenkomplexe stehen im Mittelpunkt des Röntgenkongresses 2012?

Helmberger: Zum einen ist es die Abdominaldiagnostik mit ihrem interdisziplinären Anspruch und auch ihren Überschneidungen zur Onkologie einerseits und zur interventionellen Radiologie andererseits. Als weiteren Höhepunkt sehe ich die Rheumabildgebung an, die wir gemeinsam mit Rheumatologen, Nuklearmedizinern und Pädiatern diskutieren wollen. Und natürlich werden die neusten technischen Fortschritte in der Hybridbildgebung einen breiten Raum einnehmen, genauso wie die Klassiker jedes Röntgenkongresses aus der Neuroradiologie und der Skelettdiagnostik.

? Welche Neuerungen im Programm können die Teilnehmer im Vergleich zu den Vorjahren erwarten?

Helmberger: Neben der starken Interdisziplinarität, die sich in Spezial-Refresherkursen widerspiegelt, werden z.B. die kinderradiologischen Vorträge in diesem Jahr nicht ausschließlich in reinen Kinderradiologie-Sitzungen erörtert, sondern im Rahmen der thematischen Behandlung zusammen mit den Allgemeinradiologien diskutiert. Im Spezialkurs Nuklearmedizin gibt es ebenfalls eine Neuerung, denn dort werden die Vorträge jeweils von einem radiologischen und einem nuklearmedizischen Kollegen gemeinsam vorgetragen. Die Themenreihen „Fit für den Facharzt“ und „Radiologie in Klinik und Praxis“ werden über den gesamten Kongress mit vielen Veranstaltungen ausgebaut.

? Mehr als 850 Vorträge, Seminare und Workshops werden den Röntgenkongress mit Inhalt füllen. Welche Höhepunkte im wissenschaftlichen Programm dürfen auf keinen Fall verpasst werden?

Helmberger: Da möchte ich den Teilnehmern ganz besonders die Highlightvorträge unserer diesjährigen Ehrenmitglieder ans Herz legen. Diese finden im Rahmen wissenschaftlicher Sitzungen zum Schwerpunkt des jeweiligen Ehrenmit- 
glieds statt. Die Ehrenmitglieder leiten die jeweilige Sitzung auch mit ihren Vorträgen ein. Umfassender als in einer derartigen Sitzung kann man sich über ein Thema nicht auf den aktuellen Stand bringen.

? Und wenn die Seminare und Vorträge vorbei sind, erwartet die Teilnehmer ein buntes Rahmenprogramm. Auf was möchten Sie diesbezüglich besonders hinweisen?

Helmberger: Der 1. erste Abend unseres Röntgenkongresses steht ganz im Zeichen der Begegnung der Teilnehmer untereinander. Er ist erstmals als Get-together ausgerichtet und soll in lockerer Atmosphäre auf den Kongress einstimmen und viele Kontaktmöglichkeiten eröffnen. Als Ort haben wir deshalb den "Schuppen 52“, eine der derzeitigen In-Location Hamburgs, gewählt. Dieser umgebaute ehemalige Lagerschuppen liegt mitten im Hamburger Hafen und kann mit einem eigens eingerichteten Bus-Shuttle vom Kongresszentrum aus bequem erreicht werden.

? Ein wichtiger Punkt des Röntgenkongresses und auch Ihr persönliches Anliegen ist die Nachwuchsförderung. Wie wird der Röntgenkongress diesbezüglich Ihren Wünschen gerecht?

Helmberger: Da gibt es, das vor 2 Jahren begonnene Programm „Die hellsten Köpfe für die Radiologie“ und das werden wir in bewährter Weise fortsetzen und ausbauen. Für die Studenten steht dabei neben einer Studenten-Lounge auch wieder ein eigenes Programm am Samstag bereit. Und so freuen wir uns auch in diesem Jahr auf eine große Zahl studentischer Besucher.
? Die steigende Teilnehmerzahl an MTRAs spricht für die Attraktivität des Röntgenkongresses für Medizinisch-technische Radiologieassistentinnen und -assistenten. Auf was können sich die MTRAs dieses Jahr besonders freuen?

Helmberger: Das Programm der MTRA, das wiederum vom VMTB vorbereitet wurde, spiegelt ebenfalls das Motto des Kongresses und die Breite unseres Faches wider. Es geht neben diesen rein radiologischen Themen auch auf Qualitätsmanagement, Strahlenschutz und Hygieneanforderungen in der Radiologie ein. Dieses letzte ist ein Thema, das zunehmend auch in den Fokus der Öffentlichkeit gerät.

Was mir aber besonders wichtig ist, ist dass unsere Jahrestagungen wieder gemeinsam stattfinden und so der Austausch untereinander gut möglich ist.

? Auch in diesem Jahr wird die Industrieausstellung viel Spannendes für die Kongressteilnehmer bereithalten. Welche Neuerungen sind dieses Jahr zu erwarten? Helmberger: Wir freuen uns, dass auch in diesem Jahr das Industrieforum wieder prall gefüllt ist mit Bewährten, mit Neuem und sicherlich mit ganz Neuem. So viel kann man schon verraten, dass sich die Unternehmen eine Menge haben einfallen lassen und der Besuch damit allemal sehr lohnend sein wird.

Offiziell eröffnet wird die Ausstellung mit dem Kongressrundgang am Donnerstagmorgen durch eine Delegation der Deutschen Röntgengesellschaft.
? Was ist Ihr persönliches Highlight des Röntgenkongresses in Hamburg?

Helmberger: Neben dem Get-together am Mittwoch, auf das schon hingewiesen wurde, sollte man 2 Dinge während der Kongresstage meines Erachtens keinesfalls versäumen. Zum einen die Eröffnungsveranstaltung am Donnerstagmorgen nach dem 1. Refresher-Block und dann die Röntgenvorlesung am Freitag von Prof. Richard Baron aus Chicago zum Thema „The evolution of liver imaging the search for the holy grail“. Dies ist sicherlich ein sehr vielversprechendes Thema.

? Abschließend die Frage: Was möchten Sie den Kongressteilnehmern mit auf den Weg geben?

Helmberger: Ich möchte den Kongressteilnehmern zurufen: „Kommen Sie nach Hamburg, tauchen Sie ein in die Vielfalt unseres Faches und lassen Sie sich faszinieren von Neuem, von scheinbar Widersprüchlichem und von Kuriosem. Nutzen Sie die Möglichkeit zum Austausch innerhalb der Radiologie und interdisziplinär und genießen Sie trotz allem das Flair einer wunderbaren Stadt und ihrer Gastfreundschaft".

In diesem Sinne möchte ich allen zurufen „Herzlich Willkommen zum Deutschen Röntgenkongress 2012 in Hamburg“.

Das Interview führte Dr. Claudia GampeBraig, Stuttgart. 